\title{
Comparison of six commercial ELISAs for the detection of antibodies against porcine reproductive and respiratory syndrome virus (PRRSV) in field serum samples
}

\author{
Kinga Biernacka ${ }^{\mathrm{a}}$, Katarzyna Podgórska ${ }^{\mathrm{b}}$, Anna Tyszka ${ }^{\mathrm{c}}$, Tomasz Stadejek ${ }^{\mathrm{a}, *}$ \\ a Department of Pathology and Veterinary Diagnostics, Faculty of Veterinary Medicine, Warsaw University of Life Sciences-SGGW, Nowoursynowska 159c, 02-776 \\ Warsaw, Poland \\ b Department of Swine Diseases, National Veterinary Research Institute, Partyzantow 57, 24-100 Pulawy, Poland

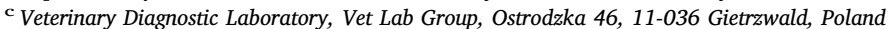

\section{A R T I C L E I N F O}

\section{Keywords:}

PRRSV

ELISA

Swine

Serum

Comparison

\begin{abstract}
A B S T R A C T
Porcine reproductive and respiratory syndrome (PRRS) is one of the most common infectious diseases of swine globally. Since the course of PRRS virus (PRRSV) infection is subclinical, laboratory diagnosis is necessary to detect the virus or specific antibodies. The aim of this study was to assess the sensitivity and specificity of IDEXX PRRS X3 Ab Test (IDEXX, USA), Civtest Suis E/S (Hipra, Spain), INgezim PRRS 2.0 (Ingenasa, Spain), VetExpert PRRS Ab ELISA 4.0 (BioNote, Korea), Pigtype PRRSV Ab (Qiagen, Germany) and PrioCHECK PRRSV Antibody ELISA (ThermoFisher, USA), using serum samples obtained from 5 conventional PRRSV-positive and 5 PRRSVnegative Polish pig farms.

Specificity of ELISAs ranged from $94.2 \%$ (ThermoFisher) to $100 \%$ (IDEXX and Hipra). ThermoFisher ELISA had the highest detection rate and detected $67.2 \%$ samples from PRRSV-positive farms as positive but considering its low specificity some of the positive results may be incorrect. IDEXX ELISA considered as a reference detected $64.8 \%$ positive sera in PRRSV-positive farms. On the other hand Hipra Elisa identified only $51.8 \%$ of samples as positive. The diagnostic sensitivity of five ELISAs relative to IDEXX ranged from 80.3\% (Hipra) to 96.3\% (ThermoFisher).

Our study showed significant differences in specificity and diagnostic sensitivity between the compared kits. The differences in the performance appeared to be practically negligible on farms where early infection with PRRSV occurred. However, on PRRSV-negative farms, or farms with PRRSV stable sow herds, some ELISAs can give results not reflecting the infection status in specific age groups.
\end{abstract}

\section{Introduction}

Porcine reproductive and respiratory syndrome (PRRS) is one of the most common and economically important infectious diseases of swine globally. The disease causes reproductive failure in breeding herds, mainly premature parturitions, late abortions and farrowing of stillborn and non-viable piglets. Respiratory symptoms can develop predominantly in younger pigs (Zimmerman et al., 2012). The disease is caused by PRRS viruses which currently are considered two different species in Arteriviridae family: PRRSV-1 and PRRSV-2 (ICTV International Committee on Taxonomy of Viruses, 2018). Both species of PRRSV are globally spread and endemic in many countries, but PRRSV1 predominates in Europe while PRRSV-2 is most common in Americas and Asia (Balka et al., 2018; Brar et al., 2015). Infection with PRRSV also predisposes to other respiratory diseases, including porcine respiratory disease complex (PRDC) (Opriessnig et al., 2011). The virus was never reported from Australia, New Zealand, Norway, Finland, Argentina, Brazil, Ecuador, Paraguay, and Uruguay (OIE, World Organisation of Animal Health. World Animal Health Information Database (WAHIS) Interface) (OIE, 2018). Sweden and Switzerland experienced outbreaks that were rapidly contained and eradication of the virus was achieved shortly after its introduction (Carlsson et al., 2009; Nathues et al., 2016).

Since clinical signs of PRRS are not characteristic and sometimes the course of PRRSV infection is subclinical, laboratory diagnosis is necessary to detect the virus or specific antibodies (Mengeling and Lager,

\footnotetext{
* Corresponding author at: Warsaw University of Life Sciences, Faculty of Veterinary Medicine, Department of Pathology and Veterinary Diagnostics, Nowoursynowska 159C, 02-776 Warsaw, Poland.

E-mail addresses: katarzyna.podgorska@piwet.pulawy.pl (K. Podgórska),wdl.serologia@vetlabgroup.pl (A. Tyszka), tomasz_stadejek@sggw.pl (T. Stadejek).
} 
2000). Sometimes so-called ELISA, or PCR farm profiles are performed using samples from different age groups of pigs to assess the virus circulation in the population. The results may assist in planning of PRRSV control programs, or evaluation of their progress, based on the changes in the virus or specific antibodies prevalence in specific groups. Currently, polymerase chain reaction (PCR) and enzyme-linked immunosorbent assay (ELISA) are most widely used for this purpose. In countries free from PRRSV serological monitoring of pig population and imported animals is practiced.

In several countries regional programs of PRRSV elimination are in place, while in Hungary a state-controlled eradication program is currently running (Olasz et al., 2016). Serological monitoring plays a crucial role in all these initiatives. At present several ELISAs are commercially available, IDEXX PRRS X3 Ab Test (IDEXX, USA) being the most widely used and generally reckoned to be the de facto gold standard of the ELISAs for detection of antibodies against PRRSV (Zimmerman et al., 2012; Sattler et al., 2014). However, ELISA results are sometimes inconclusive and require additional tests (Antunes et al., 2015).

There are several peer-reviewed publications showing generally good agreement between PRRSV ELISAs, but in many cases the evaluation was made using samples from experimentally infected, or vaccinated animals (Diaz et al., 2012; Gerber et al. 2014; Sattler et al., 2015, 2014; Seo et al., 2015; Sipos et al., 2009). Moreover, new products are being marketed, or improved versions of known ELISAs are being introduced. So, updated information on the performance of currently available kits is highly demanded.

The aim of this study was to assess the diagnostic sensitivity and specificity of six commercial PRRSV ELISA kits using serum samples obtained from 10 conventional Polish pig farms.

\section{Material and methods}

\subsection{Serum samples}

In total, 392 serum samples from individual pigs were included in the study. For the specificity comparison of ELISAs, 139 sow sera from five PRRSV-naive farms (N1, N2, N3, N4, and N5) were used (Table 1). Additionally, the collected sera were tested with real-time PCR (EZPRRSV $^{\mathrm{TM}}$ MPX 4.0, Tetracore) and proved to be free from PRRSV RNA. The herds were classified as naive based on historical and current laboratory monitoring data generated with IDEXX PRRS X3 Ab Test (IDEXX, USA) and PrioCHECK ${ }^{\circledR}$ PRRSV rtRT-PCR kit (Prionics AG, Zürich, Switzerland), as well as internally validated in house rtRT-PCR, which showed no indication of PRRSV infection from 6 months before to at least 2 years after the samples for the purpose of this study were collected.

For the diagnostic sensitivity assessment, inferred from the proportion of seropositive samples, 253 sera were obtained from five

Table 1

Summary on the samples used in the study. The age groups where PRRSV was detected in real-time PCR are marked with an asterisk.

\begin{tabular}{|c|c|c|c|}
\hline Farm & Animals & \multicolumn{2}{|c|}{ No. of samples } \\
\hline \multicolumn{4}{|c|}{ PRRSV-negative farms } \\
\hline N 1 & Sows & 30 & 139 \\
\hline N 2 & Sows & 50 & \\
\hline N 3 & Sows & 15 & \\
\hline N 4 & Sows & 20 & \\
\hline N 5 & Sows & 24 & \\
\hline \multicolumn{4}{|c|}{ PRRSV-positive farms } \\
\hline P 6 & Pigs 5, 9, 13, 17 weeks old & 48 & 253 \\
\hline P 7 & Pigs 5*, 9*, 13*, 17* weeks old & 48 & \\
\hline P 8 & Pigs 5*, 9*, 13*, 17* weeks old & 63 & \\
\hline P 9 & Pigs $4,8,12,14,18^{*}$ weeks old & 46 & \\
\hline P 10 & Pigs $6,10^{*}, 14^{*}, 18^{*}$ weeks old & 48 & \\
\hline
\end{tabular}

PRRSV- positive farms (P6, P7, P8, P9 and P10). The samples were collected every 3-4 weeks, from about 5 to 18 weeks of age, from the same groups of pigs (longitudinal sampling) (Table 1). The positive status of the farms was identified on the basis of the medical and diagnostic history, as well as through the real-time PCR testing (EZPRRSV $^{\mathrm{TM}}$ MPX 4.0, Tetracore) of the collected samples. In all PRRSVpositive farms, except for P6, PRRSV-1 RNA was detected in growing or fattening pigs (Table 1 ).

\subsection{ELISA kits}

All the serum samples were tested with six indirect ELISAs commercially available in Poland: IDEXX PRRS X3 Ab Test (IDEXX, USA), Civtest Suis E/S (Hipra, Spain), INgezim PRRS 2.0 (Ingenasa, Spain), VetExpert PRRS Ab ELISA 4.0 (BioNote, Korea), Pigtype PRRSV Ab (Qiagen, Germany) and PrioCHECK PRRSV Antibody ELISA (ThermoFisher, USA). In Hipra ELISA, plates are coated with the specific PRRSV-1 antigen-glycoprotein rich extract (Sattler et al., 2015), while in the remaining kits the antigen consists of recombinant PRRSV1 and PRRSV-2 nucleocapsid proteins. Thus, all ELISAs, except for Hipra, detect antibodies to both PRRSV species. All assays were performed according to the manufacturers' recommendations. Initial sample dilution was 1:20 for ThermoFisher, 1:40 for IDEXX, Ingenasa, BioNote and Qiagen and 1:200 for Hipra.

Sample to positive (S/P) ratios in IDEXX, Ingenasa, BioNote and Qiagen ELISAs were calculated using the following formula: S/ $\mathrm{P}=$ (optical density (OD) of sample - OD of negative control)/(OD of positive control - OD of negative control). In IDEXX, Ingenasa, BioNote and Qiagen ELISAs, sample to positive (S/P) cut-off value is set at 0.4. In ThermoFisher ELISA the cut-off value is set at $30 \%$ positivity (PP), calculated according to a similar formula (the result is multiplied by 100 ) and corresponding to S/P 0.3. In Hipra ELISA the cut-off is set at 20 relative index $\mathrm{x} 100$ (IRPC), which formula is also similar to the aforementioned and corresponds to S/P 0.2. For the sake of clarity, the results obtained with all ELISAs used in the study were expressed as $\mathrm{S} / \mathrm{P}$ ratios.

In case of unexpected results (e.g. positive results in naive farms, inconsistent results for a sample from positive farms obtained with different kits) samples were re-tested up to two times (three times in total). Also, the testing was repeated in case of doubtful (just below the cut-off) results. The final status of the sample was defined based on the majority of the obtained results in the repeated tests.

\subsection{Evaluation of ELISA kits}

The specificity of the ELISAs was assessed using truly negative serum samples from sows from five farms. The diagnostic sensitivity of the ELISAs was determined using the samples from five PRRSV-positive farms. As the pigs represented different age groups, and the true PRRSV status of individual animals was unknown, the proportion of pigs reacting positively in each ELISA was compared. Also, the profiles of seroconversion against PRRSV were determined and compared using different kits. Next, the diagnostic sensitivity of ELISAs was compared to IDEXX since this test is considered globally a reference commercial kit (Zimmerman et al., 2012). For this comparison, the serum samples positive in IDEXX ELISA were presumed to have a true positive status.

The general agreement of the tested ELISAs with IDEXX was determined with the kappa coefficient. Additionally, the proportion of consistent results between tests was evaluated based on the Cochran $\mathrm{Q}$ test to measure the significance of a disagreement between methods. Repeated Measures ANOVA with the post-hoc HSD Tukey test was applied to compare mean S/P ratios of IDEXX ELISA and the other kits in PRRS-positive farms, and within specific age groups and farms according to the infection pattern (Statistica 13.0, TIBCO, USA). The level of statistical significance was set at $p<.05$. 
Table 2

The number and percentage of positive results obtained with all ELISAs in the analysis of serum samples from PRRSV-negative farms.

\begin{tabular}{|c|c|c|c|c|c|c|c|c|c|c|c|c|c|}
\hline \multirow[t]{2}{*}{ ELISA } & \multicolumn{2}{|c|}{ N1 (30 samples) } & \multicolumn{2}{|c|}{ N2 (50 samples) } & \multicolumn{2}{|c|}{ N3 (15 samples) } & \multicolumn{2}{|c|}{ N4 (20 samples) } & \multicolumn{2}{|c|}{ N5 (24 samples) } & \multicolumn{3}{|c|}{ Total number of samples } \\
\hline & No. & $\%$ & No. & $\%$ & No. & $\%$ & No. & $\%$ & No. & $\%$ & Tested & Positive & $\%$ \\
\hline IDEXX & 0 & 0.0 & 0 & 0.0 & 0 & 0.0 & 0 & 0.0 & 0 & 0.0 & 139 & 0 & 0.0 \\
\hline Hipra & 0 & 0.0 & 0 & 0.0 & 0 & 0.0 & 0 & 0.0 & 0 & 0.0 & 139 & 0 & 0.0 \\
\hline Ingenasa & 2 & 6.7 & 2 & 4.0 & 0 & 0.0 & 1 & 5.0 & 1 & 4.2 & 139 & 6 & 4.3 \\
\hline BioNote & 0 & 0.0 & 0 & 0.0 & 1 & 6.7 & 0 & 0.0 & 1 & 4.2 & 139 & 2 & 1.4 \\
\hline Qiagen & 0 & 0.0 & 1 & 2.0 & 0 & 0.0 & 0 & 0.0 & 1 & 4.2 & 139 & 2 & 1.4 \\
\hline ThermoFisher & 0 & 0.0 & 6 & 12.0 & 0 & 0.0 & 0 & 0.0 & 2 & 8.3 & 139 & 8 & 5.8 \\
\hline
\end{tabular}

\section{Results}

\subsection{Specificity of the ELISA kits}

The assessment of the specificity of the six ELISAs using 139 truly negative serum samples from sows from five farms, identified 33 situations when one of 16 serum samples reacted positive, or slightly under the cut off, with one or more ELISA kits. These results were considered suspicious and each test, result of which was suspected to be unspecific, was repeated. One, 2, 7, 4, 3 and 16 serum samples required re-testing with IDEXX, Hipra, Ingenasa, Qiagen, BioNote and ThermoFisher ELISAs, respectively. Repeating of these tests confirmed the original positive, or slightly under the cut off result, in 27 cases. Only 1, 2 and 3 sera tested with Qiagen, Hipra and ThermoFisher, respectively, changed their status from positive to negative.

In summary, all serum samples from PRRSV negative farms reacted negatively with IDEXX and Hipra ELISAs (100\% specificity). Ingenasa, BioNote, Qiagen and ThermoFisher ELISAs provided 6, 2, 2 and 8 falsepositive results, which corresponded to $96.6 \%, 98.6 \%, 98.6 \%$ and $94.2 \%$ specificity, respectively (Table 2 ). Thus, ThermoFisher ELISA was found to be the least specific in this analysis.

Interestingly, S/P ratio obtained with Ingenasa ELISA for sample 11 from farm N2, classified as false positive, was surprisingly high, reaching 2.23 (Fig. 1). S/P ratios obtained for that sample in the other ELISAs were between 0.000 and 0.067 , so well below the cut-off value. Also, sample 21 from farm N5 reacted highly positive with Ingenasa ELISA (S/P 1.69). BioNote, Qiagen and ThermoFisher ELISAs recognized the same serum sample as positive.

In total, false-positive results were obtained in four out of five PRRSV-negative herds. For ThermoFisher ELISA, the distribution of false-positive results was restricted to two farms. Six positive results were found in farm N2 and two positive results in farm N5.

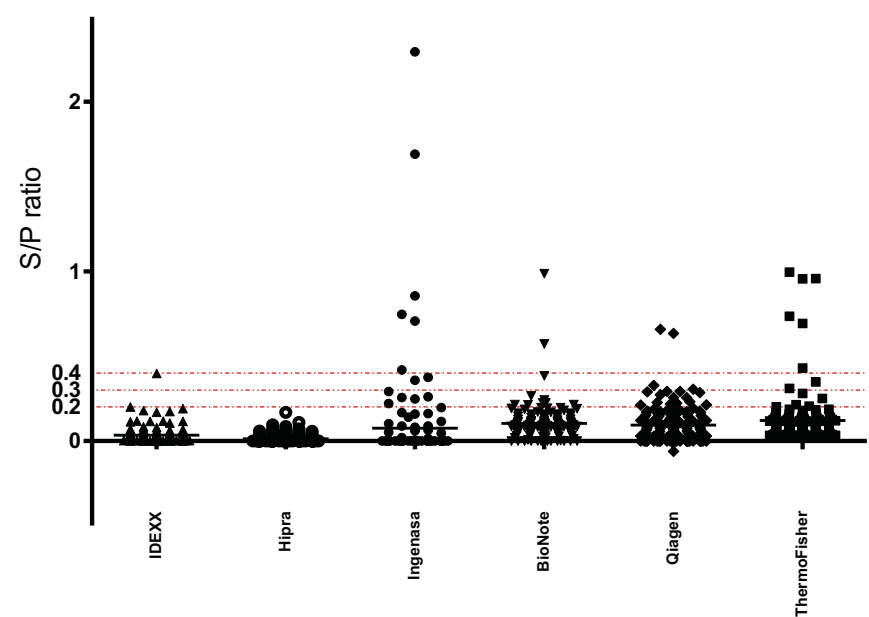

Fig. 1. S/P ratios obtained with different ELISAs in sow serum samples from PRRSV-negative farms.

\subsection{Diagnostic sensitivity of the ELISA kits}

Because the true PRRSV infection status of the animals sampled from five positive farms was unknown, the diagnostic sensitivity of the six ELISAs was assessed based on the comparison of the proportion of samples from those farms that reacted positively. In total, IDEXX, Hipra, Ingenasa, BioNote, Qiagen and ThermoFisher ELISAs detected 64.8\%, $52.2 \%, 59.3 \%, 56.9 \%, 63.6 \%$ and $67.2 \%$ of positive samples from these farms, respectively (Table 3). Thus, ThermoFisher ELISA detected the highest proportion of seropositive samples in PRRSV-positive farms, followed by Qiagen and IDEXX ELISAs.

Additionally, the sensitivity of the five ELISAs was compared to the IDEXX test, generally accepted as a gold standard in PRRS ELISA, taking into account only the samples, which reacted positively in the latter ELISA, considered here as truly positive. The sensitivity relative to IDEXX was: $80.3 \%, 89.6 \%, 86.0 \%, 95.1 \%$ and $96.3 \%$ for Hipra, Ingenasa, BioNote, Qiagen and ThermoFisher ELISAs, respectively (Table 4). In this comparison, Qiagen and ThermoFisher ELISAs performed much closer to IDEXX than the remaining ELISAs.

\subsection{Agreement between the ELISA kits}

The performance agreement of the tested ELISAs with IDEXX ELISA considered here as a reference, measured by kappa coefficient was good to very good and ranged between 0.757 (Hipra), 0.786 (BioNote), 0.832 (Ingenasa), 0.842 (ThermoFisher) to 0.888 (Qiagen). More in-depth analysis of the proportion of consistent results based on the Cochran $\mathrm{Q}$ test showed that there was a significant difference in the performance of IDEXX, Ingenasa and Qiagen kits compared to Hipra, BioNote and ThermoFisher ELISAs (Fig. 2).

Mean S/P ratios for the positive sera from PRRSV-positive farms differed significantly between the kits (Figs. 2, 3). The analysis of aggregated S/P ratios from all PRRSV-positive farms for each ELISA kit revealed two homogenous groups. Mean $S / P$ values obtained with Hipra and ThermoFisher were significantly lower compared to IDEXX, Ingenasa, BioNote and Qiagen ELISAs (Fig. 3A).

The performance of different ELISA kits differed depending on the stage of infection. In age groups where only maternal antibodies were present (farm P6-5 weeks of age, P9-4 weeks of age and P10-6 weeks of age) Qiagen and ThermoFisher kits gave results comparable to IDEXX, while there was a significant difference between Hipra, Ingenasa and BioNote compared to IDEXX (Cochran Q test, $p<.05$ ). The analysis of mean $\mathrm{S} / \mathrm{P}$ ratios in the same groups gave consistent results showing significantly lower mean values in Hipra, Ingenasa and BioNote ELISAs compared to IDEXX (Fig. 3B). A similar analysis of mean $\mathrm{S} / \mathrm{P}$ ratios in age groups where an early stage of infection was confirmed by RT-PCR (farm P7 and P8-5 week of age, farm P10-10 weeks of age) showed significantly higher value in IDEXX compared to all other ELISAs (Fig. 3C). However, in case of qualitative agreement measured by Cochran Q test, only Hipra's ELISA performance was significantly different compared to IDEXX. At a later stage of infection in the same farms (farm P7 and P8-9 week of age, farm P10-14 weeks of age) no difference in the performance of ELISA kits was observed. However, mean S/P ratios 
Table 3

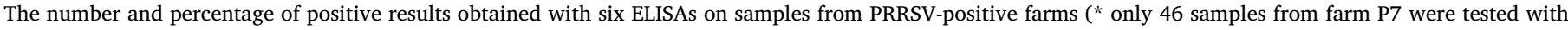
Hipra ELISA).

\begin{tabular}{|c|c|c|c|c|c|c|c|c|c|c|c|c|c|}
\hline \multirow[t]{2}{*}{ ELISA } & \multicolumn{2}{|c|}{ P6 (48 samples) } & \multicolumn{2}{|c|}{ P7 (48* samples) } & \multicolumn{2}{|c|}{ P8 (63 samples) } & \multicolumn{2}{|c|}{ P9 (46 samples) } & \multicolumn{2}{|c|}{ P10 (48 samples) } & \multicolumn{3}{|c|}{ Total number of samples } \\
\hline & No. & $\%$ & No. & $\%$ & No. & $\%$ & No. & $\%$ & No. & $\%$ & Tested & Positive & $\%$ \\
\hline IDEXX & 6 & 12.5 & 45 & 93.8 & 62 & 98.4 & 19 & 41.3 & 32 & 66.7 & 253 & 164 & 64.82 \\
\hline Hipra & 1 & 2.0 & 36 & 78.2 & 56 & 88.9 & 7 & 15.2 & 310 & 62.5 & 251 & 130 & 51.79 \\
\hline Ingenasa & 1 & 2.0 & 45 & 93.8 & 58 & 92.1 & 15 & 32.9 & 31 & 65.9 & 253 & 150 & 59.29 \\
\hline BioNote & 2 & 4.2 & 45 & 93.8 & 58 & 92.1 & 10 & 22.5 & 29 & 60.4 & 253 & 144 & 56.92 \\
\hline Qiagen & 3 & 6.3 & 46 & 95.8 & 60 & 95.2 & 18 & 39.1 & 34 & 70.8 & 253 & 161 & 63.64 \\
\hline ThermoFisher & 9 & 18.8 & 44 & 93.7 & 62 & 98.4 & 20 & 43.5 & 35 & 72.9 & 253 & 170 & 67.19 \\
\hline
\end{tabular}

Table 4

The comparison of the results obtained with ELISA kits, on samples from PRRSV-positive farms.

\begin{tabular}{|c|c|c|c|c|c|}
\hline & & \multicolumn{2}{|c|}{ IDEXX } & \multirow[t]{2}{*}{ Total } & \multirow[t]{2}{*}{ Sensitivity relative to IDEXX (\%) } \\
\hline & & Pos & Neg & & \\
\hline \multirow[t]{2}{*}{ Hipra } & Pos & 130 & 0 & 131 & 80.3 \\
\hline & Neg & 32 & 89 & 120 & \\
\hline Total & & 162 & 89 & 251 & \\
\hline \multirow[t]{2}{*}{ Ingenasa } & Pos & 147 & 3 & 151 & 89.6 \\
\hline & Neg & 17 & 86 & 102 & \\
\hline Total & & 164 & 89 & 253 & \\
\hline \multirow[t]{2}{*}{ BioNote } & Pos & 141 & 3 & 144 & 86.0 \\
\hline & Neg & 23 & 86 & 109 & \\
\hline Total & & 164 & 89 & 253 & \\
\hline \multirow[t]{2}{*}{ Qiagen } & Pos & 156 & 5 & 161 & 95.1 \\
\hline & Neg & 8 & 84 & 92 & \\
\hline Total & & 164 & 89 & 253 & \\
\hline \multirow[t]{2}{*}{ ThermoFisher } & Pos & 158 & 12 & 170 & 96.3 \\
\hline & Neg & 6 & 77 & 83 & \\
\hline Total & & 164 & 89 & 253 & \\
\hline
\end{tabular}

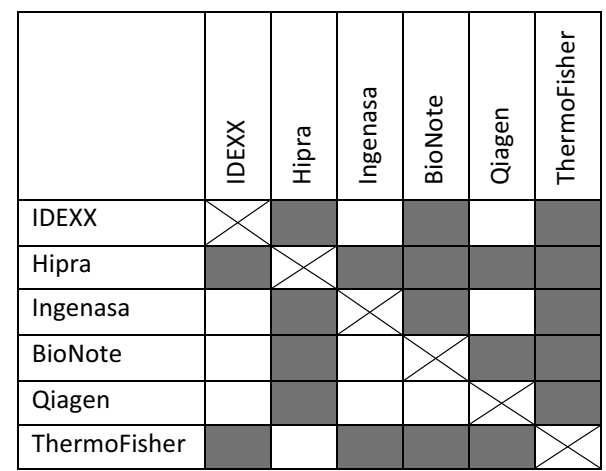

Fig. 2. Differences between ELISA kits.

Significant differences between ELISA kits (shaded) regarding a proportion of consistent results evaluated by Cochran $\mathrm{Q}$ test (upper triangle) and mean S/P ratios obtained in PRRS-positive farms evaluated by ANOVA with post-hoc Tukey's test (lower triangle). Differences were considered statistically significant at $p<.05$.

were significantly lower in Hipra and ThermoFisher kits, following the pattern observed in the overall analysis of all farms and age groups described above (Fig. 3D).

\subsection{Comparison of serological profiles of PRRSV-positive farms obtained with different ELISA kits}

As the samples tested in this study were obtained from PRRS-positive farms and represented pigs of different age, we were able to precisely compare the profiles of seroconversion obtained with different ELISAs (Supplementary Fig. 1 A, B, C, D, E). The farms represented three scenarios of PRRSV seroconversion. In farms P7 and P8 PRRSV infection was detected with PCR at 5 weeks of age and in P10 at 10 weeks of age (Table 1). An increase in the proportion of positive results following infection was evident in all ELISAs. However, the increase in mean $\mathrm{S} / \mathrm{P}$ ratios was less clearly expressed (Supplementary Fig. $1 \mathrm{~B}, \mathrm{C}$ and $\mathrm{E}$ ).

In farm P9, PCR results remained negative until 14 weeks of age and became positive at 18 weeks of age (Table 1 ). Thus, only the antibodies detected in 18 weeks old pigs can be considered active, while the antibodies in younger animals could originate only from colostrum (Supplementary Fig. 1 D). Maternal antibodies can be detected in ELISA until approximately 6 weeks of age (Chung et al., 1997). ThermoFisher ELISA detected PRRSV antibodies also at 12 and 14 weeks of age in this farm. The remaining ELISAs gave negative results in the same age groups (Supplementary Fig. $1 \mathrm{D}$ ).

In the samples from farm P6 no positive results were detected in PCR, so all seropositive pigs could possess only colostral antibodies (Supplementary Fig. 1 A). In farm P6 Hipra ELISA did not detect PRRSV antibodies at 5 weeks of age, while ThermoFisher ELISA detected antibodies in $50 \%$ of samples and IDEXX ELISA detected antibodies in $40 \%$ of samples. At 9 weeks of age in the same farm BioNote and Ingenasa ELISAs did not detect antibodies, while remaining ELISAs identified from 1 to 3 samples as positive (Supplementary Fig. 1 A).

Antibodies of maternal origin were also likely present in 5 weeks old group in farm P9 and 6 weeks old group in farm P10 (Supplementary Fig. $1 \mathrm{D}$ and E). In these age groups significantly lower performance was observed in Hipra, Ingenasa and BioNote kits compared to IDEXX, Qiagen and ThermoFisher.

\section{Discussion}

ELISA is the most widely used method for PRRSV diagnosis and monitoring on a population level. There is a number of peer-reviewed publications comparing specificity and sensitivity of commercially available kits (Diaz et al., 2012; Gerber et al., 2014; Sattler et al., 2014, 2015). However, the comparative data is often generated through the use of experimental sera. Such evaluation may not reflect the complex situation common in the field, where pigs are immunized with multiple vaccines and infected with different doses of multiple endemic viruses and bacteria, at a different age. These factors are impossible to mimic in the experimental conditions, but may impact the PRRSV antibody response detected in ELISA, and affect the decision-making process in order to control PRRS. Therefore, our study was designed to evaluate the practical performance of six PRRSV ELISAs, using sera obtained from conventional production farms.

For the specificity assessment, 139 truly negative sow sera from 5 farms were used (Table 1). False-positive results were obtained with 4 out of 6 ELISAs. The highest number of false- positives was obtained with Ingenasa (6 samples) and ThermoFisher (8 samples) ELISAs (Table 2). The results obtained with ThermoFisher ELISA for farm N2, where $12 \%$ of samples reacted false positive, were particularly puzzling. Only IDEXX and Hipra ELISAs showed no false positive results (Table 2). 
A

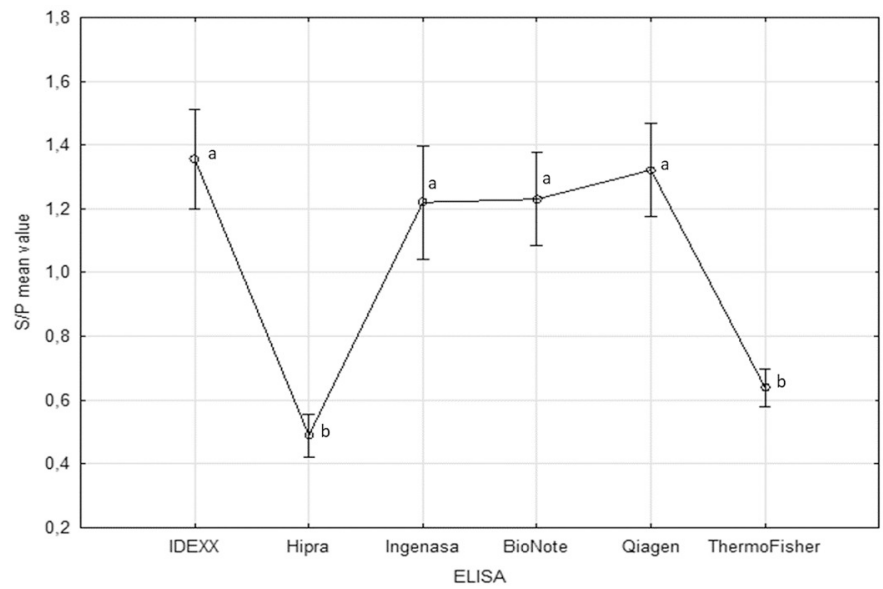

B

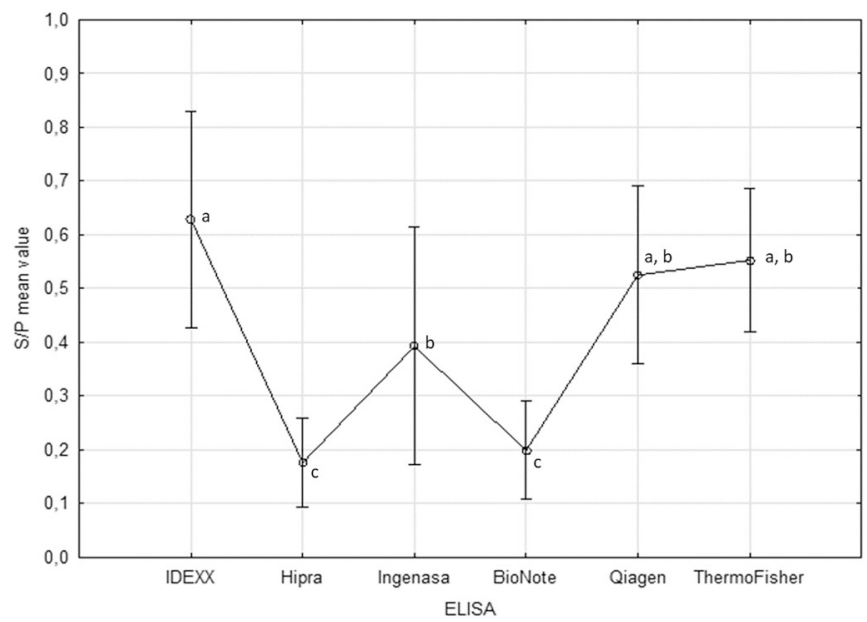

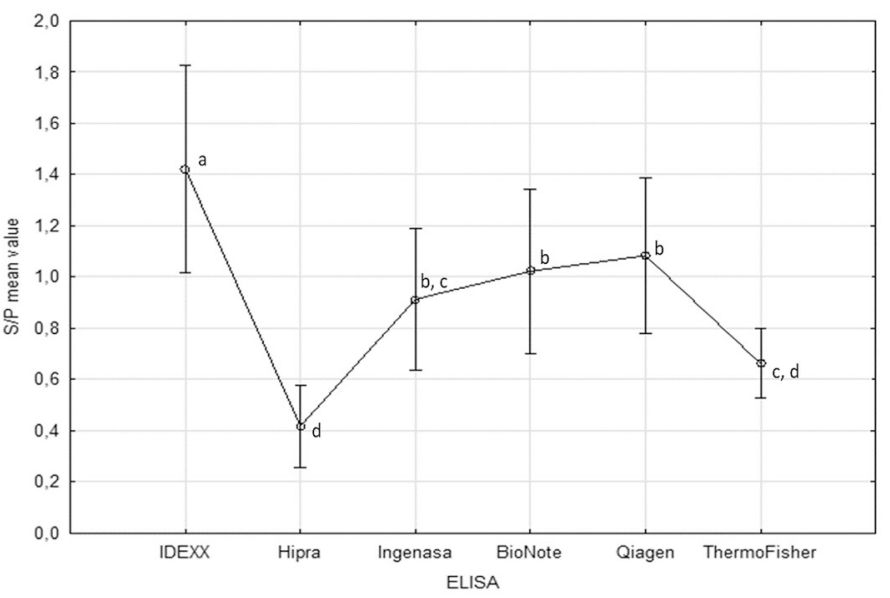

D

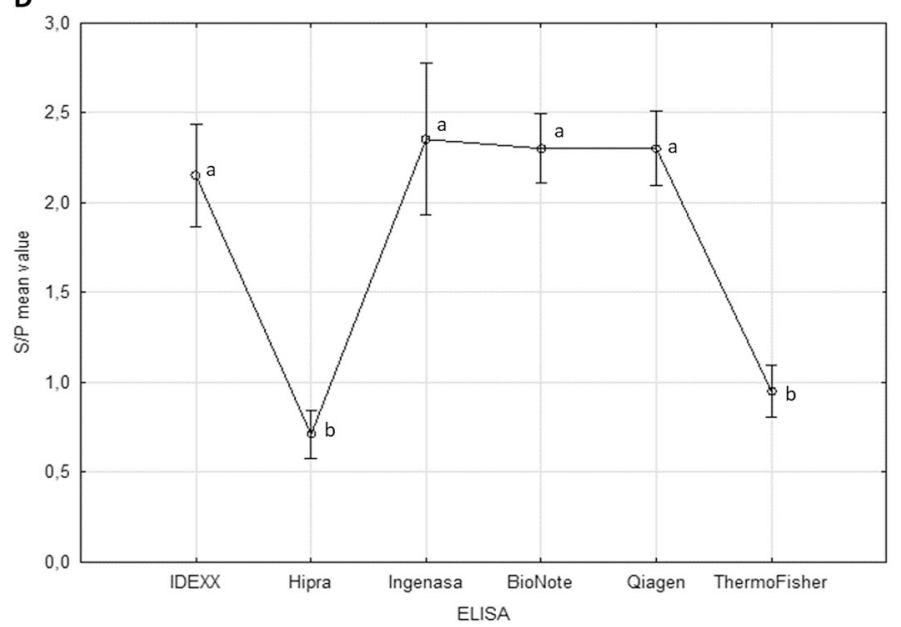

Fig. 3. Comparison of mean $S / P$ ratios of serum samples that reacted positive in each ELISA.

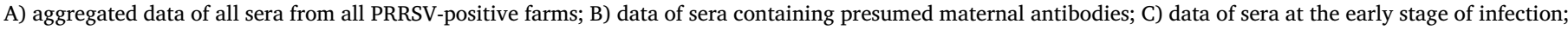

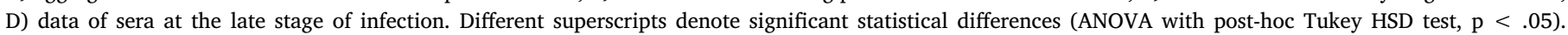
Whiskers denote 0.95 confidence intervals.

High specificity of a diagnostic kit may result in decreased sensitivity. Indeed, Hipra ELISA was the least sensitive kit evaluated in this study. It detected the lowest number of positive samples in PRRSVpositive farms (Table 3). Also, it was the least sensitive ELISA compared to IDEXX kit (Table 4). On the other hand, ThermoFisher ELISA showing the highest diagnostic sensitivity expressed as the highest proportion of positive results obtained from PRRSV-positive farms had the lowest specificity. IDEXX ELISA proved to be highly sensitive and highly specific (Tables 3 and 4). The results obtained with IDEXX ELISA, showing high specificity and sensitivity, are in agreement with the results of the earlier studies which also indicated excellent performance of this kit. The specificity comparison between IDEXX PRRS X3, INgezim PRRS 2.0, PrioCHECK PRRSV Ab Porcine and Civtest Suis PRRS E/S ELISAs performed by Sattler et al. (2015) showed results similar to ours, except for Hipra ELISA which showed only $96.4 \%$ specificity, while in our study its specificity reached $100 \%$. High specificity of Hipra ELISA was also noted by Sipos et al. (2009). Interestingly, the study of Gerber et al. (2014) showed similar sensitivity and specificity of IDEXX and Hipra ELISAs using experimental and diagnostic boar sera.

Unspecific reactions in a form of high staining background, approaching or crossing cut-off values, are often observed phenomena in ELISA and cause confusion and necessary follow-up diagnosis in monitored herds. This is particularly problematic in herds claimed free from a specific pathogen. Sometimes it occurs in individual samples from otherwise clearly negative population. The source of these reactions is often impossible to explain. The simplest explanation may be insufficient purity of an antigen used for microplate coating, or too low initial sample dilution before the test (especially in the case of relatively low cut-off value). Some authors suggested that non-specific binding of serum antibodies with some blocking agents used in ELISA production may occur (Chart et al., 1998; Konishi et al., 2010). The reason for the occurrence of false-positive results in non-competitive ELISAs may also be attributed to a phenomenon known as a bridging by heterophilic antibodies (e.g. polyspecific and idiotypic antibodies) (Schrijver and Kramps, 1998). Although the majority of false-positive results in our study occurred randomly, the detection of $12 \%$ of false positives in farm N2 with ThermoFisher may indicate the presence of specific factors contributing to the increased probability of unspecific reactions with some ELISAs, while not affecting others. In case of the ELISAs compared in the present study, the protocol of the least specific ThermoFisher ELISA required the lowest initial sample dilution and the lowest recommended low cut-off value. It is obvious that increasing cut-off and initial sample dilution would improve specificity scoring obtained from the testing of the sera used in the present study, likely at an expense of the diagnostic sensitivity score. On the other hand, the sensitivity of Hipra ELISA might be improved if lower initial sample dilution was applied. 
So-called ELISA farm profiles are assumed to reflect virus circulation in the population and may be useful for monitoring shifts in PRRSV circulation patterns in time. As the serum samples from PRRS-positive farms represented different age groups of pigs we were able to observe important differences in the serological picture of the farms, obtained with different ELISAs (Supplementary Fig. 1 A, B, C, D, E). The farms represented three scenarios of PRRSV seroconversion. In farms P7 and P8 where PRRSV infection was detected with PCR at 5 weeks of age, and in farm P10 at 10 weeks of age (Table 1), the increase in the proportion of ELISA-positive results following infection was evident with all kits (Supplementary Fig. 1 B, C and E). It can be concluded that in populations with early PRRSV infection, at 5-10 weeks of age, the differences in performance of ELISAs can be considered negligible from the practical point of view.

In farm P9 where active antibodies were detected only in 18 weeks old pigs ThermoFisher ELISA gave positive results in 12 and 14 weeks while the results from other kits were negative (Supplementary Fig. 1 D). In theory it may indicate superior sensitivity of this kit, and the ability to detect minute amounts of maternal antibodies. However, its low specificity defined based on the analysis of true negative samples rather suggests that the results obtained in 12 and 14 weeks old pigs from farm P9 may be incorrect. Moreover, highly specific and sensitive IDEXX and Qiagen ELISAs gave negative results in the same samples, which further supports that ThermoFisher ELISA results should be considered doubtful, or false positive (Supplementary Fig. $1 \mathrm{D}$ ).

Sometimes ELISA S/P ratios, or equivalent values, obtained from indirect ELISAs are used to assess the level of PRRSV specific antibodies. While superinfection of an immune population with antigenically different strain may cause an increase in the antibody levels followed by an increase in ELISA S/P ratios, comparing the results of different ELISA kits must be avoided due to significant differences in S/ $P$ values obtained with different products.

The analysis of results obtained with different ELISAs on farms with different infection status can potentially lead to different conclusions regarding PRRSV circulation, and incorrect design and evaluation of PRRSV control measures.

\section{Conclusions}

Our study showed significant differences in specificity and sensitivity between commercially available ELISA kits, despite similar antigen composition (recombinant nucleocapsid protein, except for Hipra ELISA). The differences in the performance appeared to be negligible on farms where early infection with PRRSV occurred. However, on PRRSVnegative farms, or farms with PRRSV stable sow herds, with no virus circulation, or where PRRSV infects only finishers, some ELISAs can give results not reflecting the infection status in specific age groups. Therefore, PCR diagnosis should complement ELISA diagnostic protocol to ensure the maximal reliability of obtained results. Significant differences between S/P ratios obtained with different indirect ELISAs make it impossible to directly compare such values obtained with different kits while evaluating programs of PRRS control or elimination in pig farms.

Supplementary data to this article can be found online at https:// doi.org/10.1016/j.rvsc.2018.10.005.

\section{Conflict of interest statement}

The authors declare that they have no competing interests.

Red lines correspond to cut-off $\mathrm{S} / \mathrm{P}$ ratios 0.2 (for Hipra), 0.3 (for ThermoFisher) and 0.4 (for IDEXX, Ingenasa, BioNote and Qiagen), respectively. (For interpretation of the references to color in this figure legend, the reader is referred to the web version of this article.)

\section{Acknowledgements}

This work was supported by the grant from Polish National Science Centre (NCN) [grant number 2013/11/B/NZ7/04950] and by the EU Horizon 2020 project SAPHIR [grant number 633184].

\section{References}

Antunes, A.C.L., Halasa, T., Lauritsen, K.T., Kristensen, C.S., Larsen, L.E., Toft, N., 2015 Spatial analysis and temporal trends of porcine reproductive and respiratory syndrome in Denmark from 2007 to 2010 based on laboratory submission data. BMC Vet. Res. 11, 1-11.

Balka, G., Podgórska, K., Brar, M., Bálint, Á., Cadar, D., Celer, V., Dénes, L., Dirbakova, Z., Jędryczko, A., Márton, L., Novosel, D., Petrovic, T., Sirakov, I., Szalay, D., Toplak, I., Leung, F.C.-C., Stadejek, T., 2018. Genetic diversity of PRRSV 1 in Central Eastern Europe in 1994-2014: origin and evolution of the virus in the region. Sci. Rep. 17 (8), 7811.

Brar, M.S., Shi, M., Murtaugh, M.P., Leung, F.C., 2015. Evolutionary diversification of type 2 porcine reproductive and respiratory syndrome virus. J. Gen. Virol. 96, 1570-1580.

Carlsson, U., Wallgren, P., Renstrom, L.H., Lindberg, A., Eriksson, H., Thoren, P., EliassonSelling, L., Lundeheim, N., Nörregard, E., Thörn, C., Elvander, M., 2009. Emergence of porcine reproductive and respiratory syndrome in Sweden: detection, response and eradication. Transbound. Emerg. Dis. 56, 121-131.

Chart, H., Evans, J., Chalmers, R.M., Salmon, R.L., 1998. Escherichia coli O157 serology: false-positive ELISA results caused by human antibodies binding to bovine serum albumin. Lett. Appl. Microbiol. 27, 76-78.

Chung, W.B., Lin, M.W., Chang, W.F., Hsu, M., Yang, P.C., 1997. Persistence of porcine reproductive and respiratory syndrome virus in intensive farrow-to-finish pig herds. Can. J. Vet. Res. 61, 292-298.

Díaz, I., Venteo, Á., Rebollo, B., Martín-Valls, G.E., Simon-Grifé, M., Sanz, A., Mateu, E., 2012. Comparison of two commercial enzyme- linked immunosorbent assays for the diagnosis of porcine reproductive and respiratory syndrome virus infection. J. Vet. Diagn. Investig. 24, 344-348.

Gerber, P.F., Giménez-Lirola, L.G., Halbur, P.G., Zhou, L., Meng, X., Opriessnig, T., 2014. Comparison of commercial enzyme-linked immunosorbent assays and fluorescent microbead immunoassays for detection of antibodies against porcine reproductive and respiratory syndrome virus in boars. J. Virol. Methods 197, 63-66.

International Committee on Taxonomy of Viruses (ICTV) database. https://talk. ictvonline.org/taxonomy/. Accessed 06 April 2018, 2018.

Konishi, E., Kitai, Y., Nishimura, K., Harada, S., 2010. Antibodies to bovine serum albumin in human sera?: Problems and solutions with casein-based ELISA in the detection of natural Japanese encephalitis virus infections. Jpn. J. Infect. Dis. 63, 296-298.

Mengeling, W.L., Lager, K., 2000. A brief review of procedures and potential problems associated with the diagnosis of porcine reproductive and respiratory syndrome. Vet. Res. 31, 61-69.

Nathues, C., Perler, L., Bruhn, S., Suter, D., Eichhorn, L., Hofmann, M., Nathues, H., Baechlein, C., Ritzmann, M., Palzer, A., Grossmann, K., Schüpbach-Regula, G., Thür, B., 2016. An outbreak of porcine reproductive and respiratory syndrome virus in Switzerland following import of boar semen. Transbound. Emerg. Dis. 63, e251-e261.

OIE World Animal Health Information System database. http://www.oie.int/wahis_2/ public/wahid.php/Diseaseinformation/statuslist. Accessed 06 April 2018, 2018.

Olasz, F., Bálint, Á., Balka, G., Kádár-Hürkecz, E., Zádori, Z., 2016. Porcine reproductive and respiratory syndrome and the biology of the virus - Literature review. Magy. Állatorvosok lapja 9, 523-538.

Opriessnig, T., Giménez-Lirola, L.G., Halbur, P.G., 2011. Polymicrobial respiratory disease in pigs. Anim. Health Res. Rev. 12, 133-148.

Sattler, T., Wodak, E., Revilla-Fernandez, S., Schmoll, F., 2014. Comparison of different commercial ELISAs for detection of antibodies against porcine respiratory and reproductive syndrome virus in serum. BMC Vet. Res. 10, 300.

Sattler, T., Pikalo, J., Wodak, E., Schmoll, F., 2015. Performance of ELISAs for detection of antibodies against porcine respiratory and reproductive syndrome virus in serum of pigs after PRRSV type 2 live vaccination and challenge. Porcine Health Manag. 1,19 .

Schrijver, R.S., Kramps, J.A., 1998. Critical factors affecting the diagnostic reliability of enzyme-linked immunosorbent assay formats. Rev. Sci. Tech. Off. Int. Epiz. 17, $550-561$.

Seo, B.J., Kim, H.I., Cho, H.S., Park, B.Y., Kim, W.I., 2015. Evaluation of two commercial PRRSV antibody ELISA kits with samples of known status and singleton reactors. J. Vet. Med. Sci. 78, 133-138.

Sipos, W., Lang, C., Minani, A., Fischer, L., Ritzmann, M., Schmoll, F., 2009. Comparison of two commercial ELISA systems for the detection of PRRSV-specific antibodies with a gold standard ELISA. Wien Tierärztl. Mschr. 96, 28-33 Vet Med Austria.

Zimmerman, J., Benfield, D.A., Scott, A.D., Murtaugh, M.P., Stadejek, T., Stevenson, G.W., Torremorell, M., 2012. Porcine Reproductive and Respiratory Syndrome Virus (Porcine Arterivirus). In: Zimmerman, J., Karriker, L., Ramirez, A., Schwartz, K., Stevenson, G. (Eds.), Diseases of Swine, 10th Edition. Wiley-Blackwell, pp. 461-486. 